\title{
Parallel High-Performance Computing in Geomechanics with Inner/Outer Iterative Procedures
}

\author{
Radim Blaheta, Ondřej Jakl, Jiří Starý \\ Institute of Geonics, Czech Academy of Sciences, \\ Studentská 1768, 70800 Ostrava-Poruba, Czech Republic \\ \{blaheta, jakl, stary\}@ugn.cas.cz
}

\begin{abstract}
In this paper we address the solution of large linear systems arising from the mathematical modelling in geomechanics and show an example of such modelling. The solution of linear systems is based on displacement decomposition or domain decomposition techniques with inexact solution of the arising subproblems by inner iterations. The use of inner iterations requires a generalization of the preconditioned CG method but brings additional benefits for parallel computation, possibility of reduction of the interprocessor communications and an additional tool of load balance.
\end{abstract}

\section{Introduction}

The mathematical modelling in geomechanics, mostly based on the finite element solution of boundary value problems, frequently leads to high computing requirements. These are mainly due to the following reasons:

- considering of large 3D domains for the solution of far field problems as well as for proper specification of boundary conditions induced by the virgin stress in rocks or soils,

- solution of a number of variants corresponding to the various construction stages, different input data (mostly given with a considerable uncertainty) as well as to optimization of the design.

Moreover, sometime it is necessary to model more complicated behaviour of geomaterials, time effects from rheology or dynamics as well as coupling of the mechanical phenomena to underground water flow, thermal loading etc. An example of large scale modelling is given in the next Section.

High computational requirements demand the use of powerful parallel computer systems as well as efficient numerical methods well tuned with the solved problem and the available computer. As the most laborious part of the finite element analysis is the solution of large linear systems, we shall concentrate in this paper to this topic.

For the solution of large linear systems, we suggest here to use decomposition techniques, which lead to a straightforward parallelization. Namely, we 
shall consider displacement decomposition and overlapping domain decomposition, see Section 3. For the solution of the arising subproblems, we suggest to use inner iterations, which also allow to optimize the computer time consisting from both computation and interprocessor communication and balance the load of processors. The inner iterations disturb the standard properties of the preconditioners, which should be treated in the outer iterations, see Section 4. The results from testing the described methods on a benchmark problem, which is derived from the modelling described in Section 2, are reported in the last Section.

\section{An example of large scale modelling}

As an example of large scale geomechanical problem which requires application of high-performance computing, we shall describe the assessment of the development of the stress fields during the mining at the uranium ore deposit Rožná in the Czech Republic. The deposit is situated in metamorphic sedimentary effusive rocks with uranium mineralization of hydrothermal origin situated in zones of veins arising from longitudinal faults with inclination $45^{\circ}-70^{\circ}$ to the West, see Figure 1. For the modelling, a 3D domain is selected, which has the

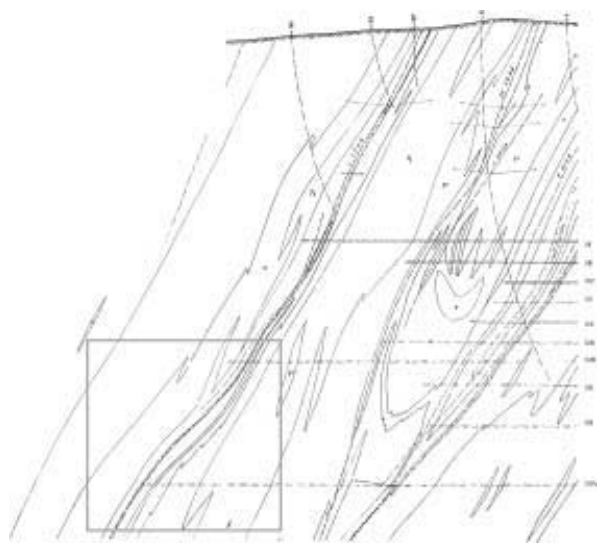

Fig. 1. (left) East-West cross-section of the deposit with 4 th zone and the modelled area in the left down corner.

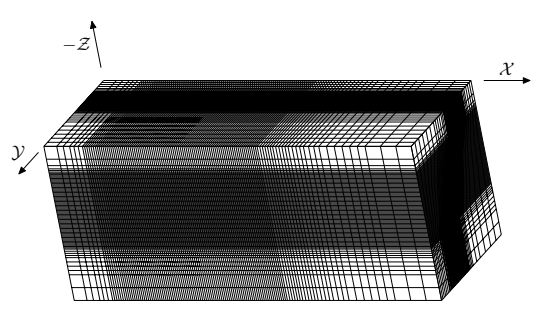

Fig. 2. FE mesh: $124 \times 137 \times 76$ nodes.

dimensions $1430 \times 550 \times 600$ meters. For the finite element modelling, this domain is divided into hexahedral bricks, which are subsequently divided, each brick into six tetrahedra. The finite element analysis with linear tetrahedral elements uses 3873264 degrees of freedom. The exploited mesh can be seen from Figure 2. The loading is given by the weight of the material and the action of the surrounding rocks existing under the pre-mining stress state. Due to the performed measurements of this pre-mining stress, the modelling was performed for two extreme cases: the isotropic pre-mining stress $\sigma_{x x}=\sigma_{y y}=\sigma_{z z}, \sigma_{x y}=\sigma_{y z}=\sigma_{z x}=0$ and the anisotropic one $\sigma_{x x}=\sigma_{y y}=1.25 \sigma_{z z}, \sigma_{x y}=0.75 \sigma_{z z}, \sigma_{y z}=\sigma_{z x}=0$. The vertical stress $\sigma_{z z}$ is given by the weight of the rocks, $\sigma_{z z}=-\gamma h$ where $\gamma$ is the averaged specific weight of the rocks, $h$ is the depth under surface. For a 
future modelling, an identification procedure was suggested in [7], which allows to reduce the uncertainties concerning this pre-mining stress state.

The modelling itself consists in solving a sequence of boundary value problems with different material distribution, which corresponds to four selected stages of mining. In these stages, we solve the equilibrium equations with the use of a proper linear constitutive relations. Such approach requires a estimate of the effective elastic moduli for the rocks, ore as well as for the damaged rocks (the goaf), which appear in the vicinity of the mine openings. The estimate of the extent of the zone of damaged material and the estimate of the material constants for the goaf is another important source of uncertainty, see e.g. the sensitivity analysis performed in [6].

As the pre-mining stress is not compatible with any apriori known displacements, the modelling sequence consists from solving four pure Neumann boundary value problems. In this case, the use of different weights of intact and damaged materials leads to some global disbalance, which can be overcome by finding generalized solution of the arising singular systems. Note also that stresses, which are of our primary interest, are unique.

The above sequence of Neumann problems (Neumann sequence) can be solved by iterative solvers. But more advantageous may be to use another sequence of problems (Dirichlet sequence), which consists from one auxiliary step with solving the pure Neumannn problem for the computation of pre-mining displacements and using these displacements as a pure Dirichlet boundary conditions in the subsequent modelling steps. Note that the maximal difference in the stress computed by these two sequences was found to be less than $7 \%$, which is acceptable for the performed modelling.

As a conclusion, we can summarize that the performed modelling requires repeated solution of large linear systems of nearly four million of unknown. Moreover, these systems can be singular.

\section{Two decomposition techniques}

The finite element analysis of boundary value problems, like the problems described in the previous Section, requires the solution of the equation

$$
A u=b,
$$

which can be interpreted as the operator equation in the finite element space $V$ or the corresponding linear system in the space of algebraic vectors $R^{n}$, where $n$ is large. For 3D analysis $n$ is typically in the range $10^{5}-10^{7}$.

The solution of (1) is usually done by the conjugate gradient (CG) method with a proper preconditioning, see e.g. [1]. The construction of preconditioning can be based on a decomposition of $V$,

$$
V=V_{1}+\ldots+V_{p},
$$

where $V_{k}$ are subspaces of $V$, which are not necessarily linearly independent. Of course, the corresponding decomposition of the algebraic space $R^{n}$ will be used for implementation of the preconditioners. 
The general scheme for the construction of space decomposition preconditioners is the following. Let

$$
R^{n} \leftrightarrow V, \quad R^{n_{k}} \leftrightarrow V_{k},
$$

$I_{k}: R^{n_{k}} \rightarrow R^{n}$ be the prolongation given by the inclusion $V_{k} \subset V$,

$$
R_{k}: R^{n} \rightarrow R^{n_{k}} \quad \text { be the restriction given by } R_{k}=I_{k}^{T} \text {. }
$$

Let $A$ be the $n \times n$ stiffness matrix and $A_{k}=R_{k} A I_{k}$ be the matrices corresponding to the subproblems on the subspaces. Note that if $A$ is symmetric positive definite then $A_{k}$ has the same properties.

Now, the preconditioner can be defined by the following algorithm for the computation of the pseudoresidual $g$ from the residual $r$. It can be interpreted as a linear mapping $G: r \mapsto g$.

$$
\begin{aligned}
& \text { Algorithm 1 } \\
& \begin{array}{l}
g=0 \\
\text { for } k=1, \ldots, p \text { do } \\
\quad g \leftarrow g+I_{k} A_{k}^{-1} R_{k} z_{k}
\end{array} \\
& \text { end }
\end{aligned}
$$

The operations

$$
w_{k}=A_{k}^{-1} v_{k}
$$

can be implemented as

$$
w_{k}=S_{k}\left(v_{k}\right),
$$

where $S_{k}\left(v_{k}\right)$ results from solving the subproblem $A_{k} w_{k}=v_{k}$ by inner iterations. The inner iterations can be given again by the CG method stopped by the condition

$$
\left\|v_{k}-S_{k}\left(v_{k}\right)\right\| \leq \varepsilon_{0}\left\|v_{k}\right\| .
$$

In the case of inexact solution of the subproblems, the mapping $G$ become nonlinear. It may require some measures to be implemented in outer iterations, see the next Section.

For more details about general space decomposition preconditioners, see e.g. [13] and the references therein. Note that in this paper, we consider only the additive preconditioners, which lead to a straightforward parallelization.

We shall use two particular decomposition techniques, the displacement decomposition (see [3], [5], [8]) and the overlapping domain decomposition (see e.g. [12] for the description). Possibly, we can also use a combination of these techniques, cf. [9].

The displacement decomposition concerns the solution of the elasticity problem in a domain $\Omega \subset R^{d}(d=2,3)$ by the finite element method with Lagrangian finite elements. In this case, the algebraic vectors from $R^{n}$ represent nodal displacements. In a special, so called separate displacement component ordering of the degrees of freedom, the vectors $v \in R^{n}$ have block form $v=\left(v_{1}, \cdots, v_{d}\right)^{T}$, 
where $v_{k}$ represents nodal displacement in $k$ - th coordinate direction of the coordinate system exploited for description of $\Omega$. Then $R^{n_{k}}$ correspond to the blocks,

$$
I_{k}: v_{k} \mapsto\left(v_{1}, \ldots, v_{d}\right)^{T}, \quad v_{l}=0 \text { for } l \neq k .
$$

This decomposition allows to construct the preconditioners, which have been studied in [3], [5], [2], [8].

In this paper, the domain decomposition concerns again the solution of elasticity problems in the domain $\Omega$, which is divided into finite elements $E \in \mathcal{T}_{h}$. The decomposition starts from decomposition of $\Omega$ into non-overlapping subdomains $\hat{\Omega}_{k}$, which are subsequently extended into overlapping subdomains $\Omega_{k}, \Omega=\bigcup_{k=1}^{p} \Omega_{k}$. We assume that each $\hat{\Omega}_{k}, \Omega_{k}$ can be represented as a union of some elements from the global division $\mathcal{T}_{h}$. Then the division of $\Omega$ induces a decomposition of the finite element space $V$ with the subspaces $V_{k}$,

$$
V_{k}=\left\{v \in V: v=0 \text { in } \Omega \backslash \Omega_{k}\right\} .
$$

In all spaces $V, V_{1}, \ldots, V_{p}$, we can use the same finite element basis functions. The isomorphism with $R^{n}, R^{n_{1}}, \ldots, R^{n_{p}}$ then allows simple construction of the prolongation represented by a Boolean matrix,

$$
I_{k}=\left[c_{i j}\right], 1 \leq i \leq n, 1 \leq j \leq n_{k}
$$

where $c_{i j}=1$ if the degrees of freedom $i$ and $j$ correspond to the same finite element basis function, otherwise $c_{i j}=0$.

The efficiency of the domain decomposition preconditioner improves with the increasing overlap, but deteriorates with the increasing number of subdomains $p$, see e.g. [12] for the explanation. This drawback can be remowed and overall efficiency can be improved by using extended decomposition with an additional subspace $V_{0}$ corresponding to discretization of the global problem with the aid of a coarser finite element division $\mathcal{T}_{H}$. If $\mathcal{T}_{h}$ is a refinement of $\mathcal{T}_{H}$, then $I_{0}$ is simply defined by the inclusion $V_{0} \subset V$. If $\mathcal{T}_{H}$ and $\mathcal{T}_{h}$ are not nested, then we have to use more complicated interpolation $I_{0}$, which may be relatively costly to create and perform. For this reason, we consider also another choice of $V_{0}$ constructed from $V$ by aggregation. This construction was introduced for multigrid methods [4], its use for the overlapping Schwarz method is analyzed e.g. in [11].

Let $\left\{\phi_{i}: i=1, \ldots, n\right\}$ is the FE basis of $V_{h}$ and let $\{1, \ldots, n\}=\bigcup_{k=1}^{N} J_{k}$ where $J_{k}$ are disjoint sets. Then the aggregation space can be defined as $V_{0}=$ $\operatorname{span}\left\{\Psi_{k}: k=1, \ldots, N\right\}, \Psi_{k}=\sum_{i \in J_{k}} \phi_{i}$. In this case, we can again construct a Boolean prolongation $I_{0}: R^{N} \rightarrow R^{n}$, which also allows a cheap construction of the matrix $A_{0}=\left(I_{0}\right)^{T} A I_{0}$.

\section{GPCG method}

The use of inner iterations for solving the subproblems leads to generally nonlinear preconditioner $G$, which approximates the linear space decomposition preconditioner defined by the same decomposition and exact solution of subproblems. Such nonlinear preconditioner can be implemented within the standard 
CG method, but the arising preconditioned CG may be not efficient or even fail to converge. Therefore, we shall describe here a simple generalization of the CG method, which is convenient to use in this case. For more details, see [1], Chapter 12 , and [8].

Let $\langle u, v\rangle=u^{T} v$ denotes the standard inner product in $R^{n}, J(v)=\frac{1}{2}\langle A v, v\rangle-$ $\langle b, v\rangle$ be the energy functional, whose minimization is equivalent to the solution of the system (1). Then the GPCG iterations with a general preconditioner $G$ use the following steps.

GPCG [m] iteration, $1 \leq m \leq \infty$.

Given $u^{i}, v^{i}, \ldots, v^{i+1-} m_{i+1}$, where $m_{i}=\min \{i, m\}$, compute:

1. $\alpha_{i} \in R: J\left(u^{i}+\alpha_{i} v^{i}\right)=\min _{\alpha \in R} J\left(u^{i}+\alpha v^{i}\right)$

2. $u^{i+1}=u^{i}+\alpha_{i} v^{i}, r^{i+1}=r^{i}-\alpha_{i} A v^{i}$

3. $g^{i+1}=G\left(r^{i+1}\right)$

4. $v^{i+1}=g^{i+1}+\sum_{k=1}^{m_{i+1}} \beta_{i+1}^{(k)} v^{i+1-k}, \quad\left\langle A v^{i+1}, v^{i+1-k}\right\rangle=0 \quad \forall k=1, \ldots, m_{i+1}$.

The following Theorem is important for the implementation of GPCG.

\section{Theorem 1.}

1. $\alpha_{i}=\frac{\left\langle r^{i}, v^{i}\right\rangle}{\left\langle A v^{i}, v^{i}\right\rangle}=\frac{\left\langle r^{i}, g^{i}\right\rangle}{\left\langle A v^{i}, v^{i}\right\rangle}$

2. $\beta_{i+1}^{(k)}=-\frac{\left\langle g^{i+1}, A v^{i+1-k}\right\rangle}{\left\langle A v^{i+1-k}, v^{i+1-k}\right\rangle}=\frac{\left\langle g^{i+1}, r^{i+2-k}\right\rangle-\left\langle g^{i+1}, r^{i+1-k}\right\rangle}{\left\langle g^{i+1-k}, r^{i+1-k}\right\rangle}$

3. the GPCG $[\mathrm{m}]$ algorithm will not break down if $r \neq 0$ implies

$$
\langle r, g\rangle=\langle r, G(r)\rangle \neq 0 .
$$

The correctness and convergence of GPCG[m], $1 \leq m \leq \infty$, in the case of the additive space decomposition preconditioner with inexact subproblem solvers can be shown by using the following Theorem, for the proof see [8].

\section{Theorem 2.}

Let $G$ be an approximation to a SPD matrix $B$,

$$
\left\|G(r)-B^{-1} r\right\|_{B} \leq \varepsilon_{0}\left\|B^{-1} r\right\|_{B}
$$

and let $\gamma_{1}, \gamma_{2}$ be two positive constants such that

$$
\gamma_{1}\langle B v, v\rangle \leq\langle A v, v\rangle \leq \gamma_{2}\langle B v, v\rangle \quad \forall v \in R^{n}
$$

Then for $m \geq 1$ and arbitrary $\varepsilon_{0} \in\langle 0,1)$ the GPCG $[\mathrm{m}]$ method does not break down and converges,

$$
\left\|u^{i+1}-A^{-1} b\right\|_{A} \leq \sqrt{1-\left(1-\varepsilon_{o}^{2}\right) \kappa^{-1}}\left\|u^{i}-A^{-1} b\right\|_{A}, \quad \kappa=\gamma_{2} / \gamma_{1} .
$$




\section{Parallel implementation and inner/outer iterations}

The use of additive space decomposition preconditioners leads to very natural parallelization of the computation of the pseudoresiduals, when $p$ processors compute simultaneously the contributions from $p$ subspaces. Moreover, the described decomposition techniques induce also (nonoverlapping) decomposition of the global matrix and global vectors, which can be used for parallelization of the further steps in outer iterations.

There are two additional roles of inner iterations if the parallel implementation is used. Firstly, an increase of the accuracy of inner iterations makes the outer iteration more costly but reduces the number of outer iterations. This may be very advantageous if we use parallel system with relatively slow communication rate. This effect will be demonstrated in the next Section. Secondly, variable number of iterations for different subproblems can be used for better balancing the load of processors and speed-up the computations.

\section{Performance of the methods}

For numerical testing, we shall use the linear system arising from the stress computation concerning the last stage of the modelling described in Section 2 as a benchmark. In this benchmark, we use the pure Dirichlet boundary conditions with the prescribed displacement computed in a preliminary stage.

We present here results from computing on a multi-processor computer SUN HPC 6500 with processors UltraSPARC II/400, 1 GB RAM, a simple cluster of PC's with Intel Pentium III/500, 384 MB RAM and Ethernet 100 interconnection (CLUSTER-1), and more powerful cluster of PC's with AMD Athlon/1400, $768 \mathrm{MB}$ RAM and Ethernet 100 interconnection (CLUSTER-2). Note that the numerical experiments on clusters of PC's are still in progress.

\begin{tabular}{|r|c|c|c|c|}
\hline Solver & $\begin{array}{c}\text { Number of } \\
\text { iterations }\end{array}$ & $\begin{array}{c}\text { SUN } \\
\text { Time [s] }\end{array}$ & $\begin{array}{c}\text { CLUSTER-1 } \\
\text { Time [s] }\end{array}$ & $\begin{array}{c}\text { CLUSTER-2 } \\
\text { Time }[\mathrm{s}]\end{array}$ \\
\hline$P C G-G_{F}$ & 83 & 668 & 2653 & 420 \\
\hline$P C G-G_{I}$ & 11 & 653 & 918 & 326 \\
\hline$G P C G[1]-G_{I}$ & 8 & 557 & 787 & 274 \\
\hline
\end{tabular}

Table 1.

Table 1 shows the numbers of iterations and computer times from solving the benchmark problem with the aid of the additive displacement decomposition preconditioner. Parallel computations are performed on 3 processors from the described computer systems. Zero initial guess and stopping by relative accuracy $\varepsilon=10^{-4}$ is used. PCG means the use of the preconditioned conjugate gradient method with orthogonalization of the new direction to the previous one by using

$$
v^{i+1}=g^{i+1}+\beta_{i} v^{i}
$$

with standard formula

$$
\beta_{i}=\frac{\left\langle g^{i+1}, r^{i+1}\right\rangle}{\left\langle g^{i}, r^{i}\right\rangle}
$$


which was introduced already in the pioneering paper [10]. On the other hand, GPCG[1] uses the modified formula, see Theorem 1 (2.). $G_{F}$ means inexact solution of the subproblems by replacing the subproblem matrices by their incomplete factorization, $G_{I}$ uses inner CG iterations with the same incomplete factorization as inner preconditioner. The relative accuracy for the inner iterations is $\varepsilon_{0}=10^{-1}$. By numerical experiments, it was found that this value is nearly optimal.

From Table 1 we can see that more exact solution of the subproblems by inner iterations substantially decrease the number of outer iterations. This fact has a big influence when the computations are performed on a cluster with a slow interprocessor communications.

The displacement decomposition works well and in case of 3 or 4 processor computations, it is more efficient then the domain decomposition with the same number of subproblems. But the domain decomposition is scalable and this allows to get better times by using more processors, see Figure 3 .
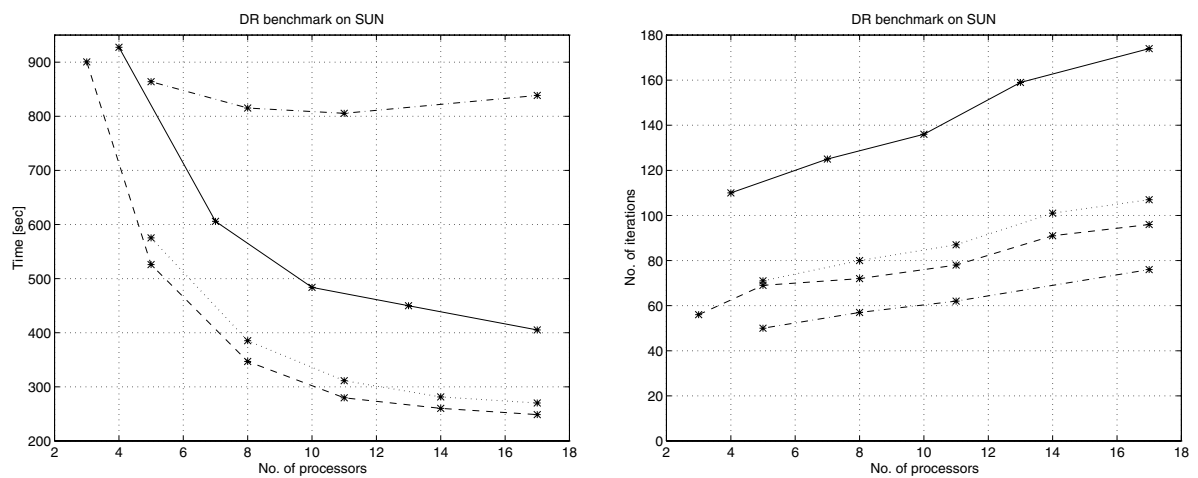

Fig. 3.

This Figure contains diagrams with numbers of outer iterations and computer time for the solution of the benchmark problem with the additive preconditioner based on domain decomposition with minimal overlap and no coarse grid problem (solid lines) and with coarse grid problem created by aggregation - grouping of $3 \times 3 \times 3$ nodes (dot-dashed), $6 \times 6 \times 6$ nodes (dashed) and $9 \times 9 \times 6$ nodes (dotted lines). The subproblems corresponding to subdomains are solved by replacing the subproblem matrices by incomplete factorization, the subproblem corresponding to the coarse grid problem is solved with relative accuracy $\varepsilon_{0}=10^{-1}$ by inner PCG iterations with the same incomplete factorization preconditioning. The number of processors is equal to the number of subproblems.

From this Figure, we can see that aggregation helps, but relatively rough aggregation was required for decrease of the computing times. Finer aggregation reduced the number of outer iterations but lead to a worse load balance of the processors. In this respect, we will try to further optimize the performance as well as test the method on clusters of PC's.

Note also that the displacement decomposition and domain decomposition can be combined, see [9]. 


\section{Concluding remarks}

In this paper, we consider inner/outer iteration processes, which arise from using space decomposition preconditioning with inexact solution of the subproblems by inner iterations.

The space decomposition is used for parallelization of the computations. Moreover, the inner/outer iterations can be used for balancing the ratio between the amount of communications (the increase of the number of inner iterations can decrease the number of outer iterations and the amount of communications among the processors). Different numbers of inner iterations for different subproblems can keep all the processors busy and speed up the solution process.

Two special decomposition techniques for solving elasticity problems have been used, the displacement decomposition and domain decomposition. For outer iterations, we used recently developed generalized preconditioned CG method. Further numerical experiments with balancing parallel computations by controlling the accuracy of inner iterations are still in progress.

\section{Acknowledgement}

The presented work is supported by the grant GAČR 105/99/1229 of the Grant Agency of the Czech Republic and the grant S3086102 of the Czech Academy of Sciences.

\section{References}

1. Axelsson, O.: Iterative Solution Methods. Cambridge University Press, 1994

2. Axelsson, O.: On iterative solvers in structural mechanics; separate displacement orderings and mixed variable methods. Mathematics and Computers in Simulation 50 (1999), 11-30

3. Axelsson, O., Gustafsson, I.: Iterative methods for the solution of the Navier's equations of elasticity. Computer Meth. Appl. Mech. Engng. 15 (1978), 241-258

4. Blaheta, R.: A multilevel method with overcorrection by aggregation for solving discrete elliptic problems. J. Comp. Appl. Math. 24 (1988), 227-239

5. Blaheta, R.: Displacement Decomposition - Incomplete Factorization Preconditioning Techniques for Linear Elasticity Problems. Num. Lin. Alg. Appl. 1 (1994), 107-128

6. Blaheta, R., Kohut, R.: Uncertainty and sensitivity analysis in problems of geomechanics. Report DAM 2000/1, IGAS Ostrava, 2000, 16pp. (in Czech)

7. Blaheta, R.: Identification methods for application in geomechanics and materials science. Report DAM 2000/2, IGAS Ostrava, 2000, 29pp.

8. R. Blaheta: GPCG-generalized preconditioned $C G$ method and its use with nonlinear and nonsymmetric displacement decomposition preconditioners. Report DAM 2001/2, Institute of Geonics AS CR, Ostrava, 2001 (submitted).

9. Blaheta, R., Byczanski, P., Jakl, O., Starý, J.: Space decomposition preconditioners and their application in geomechanics. Math. Computer Simulation, submitted

10. Hestenes, M.R., Stiefel, E.: Methods of conjugate gradients for solving linear systems. Journal of Research of the National Bureau of Standards 49 (1952), 409-436 
11. Jenkins, E.W., Kelley, C.T., Miller, C.T., Kees, C.E.: An aggregation-based domain decomposition preconditionerfor groundwater flow. SIAM J. Sci. Comput. 23 (2001), 430-441

12. Smith, B.F., Bjørstad, P.E., Gropp, W.D.: Domain Decomposition Parallel Multilevel Methods for Elliptic Partial Differential Equations. Cambridge University Press, 1996

13. Xu, J.: The method of subspace corrections. J. Comp. Appl. Math. 128 (2001), 335-362 